\title{
Kunstlabore: Für mehr Kunst in Schulen!
}

Ein Ratgeber zur Qualität

künstlerischer Arbeit in Schulen 
